\title{
Fractionation and flux decline studies of surface-patterned nanofiltration membranes using $\mathrm{NaCl}$-glycerol-BSA solutions.
}

Melissa Rickman $^{\mathrm{a}, \dagger}$, Sajjad Maruf ${ }^{\mathrm{b}}$, Elmira Kujundzic ${ }^{\mathrm{b}}$, Robert H. Davis ${ }^{\mathrm{a}}$, Alan Greenberg ${ }^{\mathrm{b}}$, Yifu Ding $^{\mathrm{b}}$, John Pellegrino ${ }^{\mathrm{b}, *}$

${ }^{\mathrm{a} D e p a r t m e n t ~ o f ~ C h e m i c a l ~ a n d ~ B i o l o g i c a l ~ E n g i n e e r i n g ~}$

University of Colorado

Boulder, CO 80309-0596

${ }^{\mathrm{b}}$ Department of Mechanical Engineering

NSF-Industry/University Cooperative Research Center for Membrane Science, Engineering and Technology (MAST)

University of Colorado

Boulder, CO 80309-0427

*Corresponding author:

john.pellegrino@colorado.edu; +1-303-735-2631

†Current address: Phillips 66, Bartlesville, OK 74003

\begin{abstract}
One of the major challenges in applying membrane technology is how to mitigate fouling, which decreases membrane productivity and lifetime. We previously developed a technique for preparing thin-film composite (TFC) polyamide membranes with submicron-patterned surfaces (via nanoimprint lithography), using interfacial polymerization techniques consistent with those used in the commercial production of reverse osmosis membranes. Herein, we present results from crossflow permeation experiments with these patterned and non-patterned TFC membranes using aqueous $\mathrm{NaCl} /$ glycerol solutions, with and without bovine serum albumin (BSA) as a model protein foulant. The $\mathrm{NaCl} / \mathrm{glycerol} /$ water fractionation properties of these membranes were not significantly affected by the imprinting process, and their separation performance is similar to that of commercially available materials. At a low transmembrane pressure with operation likely experiencing weak concentration polarization, the permeance decline is small with both imprinted and non-imprinted membranes. At a higher transmembrane pressure, however, a rapid flux decline was observed for the non-patterned membranes but not for the patterned ones. Furthermore, the patterned membranes recovered more of their initial pure water permeance after the fouling permeation experiments. These initial findings reinforce the prospect of improved long-term fouling mitigation due to surface patterning. In particular, our results
\end{abstract}


suggest that even regular $\sim 30 \mathrm{~nm}$ protruding surface patterns increase the flux with low protein deposition and may also lead to a looser structure (and, thus, easier removal) of any deposited surface protein layer.

Keywords: fouling; nanoimprinting; thin film composite 


\section{Introduction}

Membranes are increasingly applied for seawater desalination and industrial water treatment to conserve freshwater resources. Membranes also play an integral role in the energy milieu and biorefinery separations [1], for example in processing lignocellulosic biomass hydrolysates [2, 3]. However, impurities such as salts, organics, algae, bacteria, and suspended particles can result in membrane fouling [4-6]. These challenging feed streams reduce membrane productivity and lifetime, increasing operating and replacement costs [7]. A major need is to fabricate membranes that are more resistant to fouling caused by colloidal cake formation, organic adsorption, inorganic scaling, and biofilm development.

Surfaces have been engineered to reduce bioadhesion by mimicking the skin topology of fastmoving sharks [8-13]. For example, Reddy et al. reported that E.coli adhesion onto micropatterned silicone catheters was reduced by almost 50\% versus unpatterned analogs [11]. It should be noted that such surfaces, along with those in other studies [14, 15], have been embossed with features on the micron scale. Nonetheless, we have conjectured that similar principles could be extended to membranes as a fouling mitigation strategy, with the additional hypothesis that patterned surfaces may improve "mixing" at the feed-membrane interface and subsequently reduce foulant deposition. Our studies [16-18] using ultrafiltration (UF) membranes, patterned with regular features on the order of 100s of nm, to filter either model colloidal or protein suspensions, have suggested that this conjecture appears to have merit.

However, fabrication of reverse-osmosis (RO) membranes that have controlled surface patterns presents an experimental challenge due to the fast kinetics inherent to the interfacial polymerization process widely used to fabricate these membranes. Moreover, thin-film composite (TFC) polyamide membranes have intrinsically rough surfaces, with finger-like protrusions that tend to be on the order of 10 to $100 \mathrm{~nm}$ [19]. This roughness is not controlled because it is formed randomly during the diffusion-limited interfacial polymerization process [20-22]. Prior modeling efforts by Ramon, et al. [23] have shown that, depending on the orientation of the thin-film peaks and valleys relative to the underlying substrate pores, the thinfilm roughness profile can either amplify or dampen localized, high-flux "hot spots" that could serve as adsorption or scaling initiation sites. However, it is unclear whether controlled surface 
patterns and roughness might exacerbate or alleviate membrane fouling. This question provided the impetus for the further work described herein.

Earlier, we developed a method to prepare TFC RO membranes with controlled, submicron-scale surface patterns by polymerizing a polyamide thin film on top of an imprinted ultrafiltration (UF) substrate [24]. Initial batch filtration tests in a stirred cell suggested that the surface-patterned membranes reduced concentration polarization relative to their flat counterparts, possibly due to secondary flows that result from the protruding patterns. Subsequently, Maruf et al., [25] reported more detailed studies on the relationship between interfacial polymerization and processing time on the conformal growth of the TFC layer upon the patterned substrate. Also, Weinman and Husson [26] have recently extended upon the idea of patterned NF membranes by illustrating that a commercial NF_TFC (GE-HL, based on piperazine monomer) could be directly imprinted with a Si line-and-groove nanostamp (final groove depth in the membranes was $\sim 70 \mathrm{~nm}$ ). Additionally, they measured fouling resistance against sodium alginate with patterned membranes that had been surface hydrophilized, as well as left simply patterned. Their results suggest that the combination of patterning and surface hydrophilization provided the least flux reduction over their $2 \mathrm{~h}$ experiments.

Herein, we present longer-duration (in some cases $\geq 1$ day) permeation studies of aqueous sodium chloride $(\mathrm{NaCl})$ and glycerol, with added bovine serum albumin (BSA) protein as a surrogate for the range of colloidal foulants that are often present in various feed waters. Patterned TFC membranes based on interfacial polymerization with the m-phenyldiamine monomer, atop a patterned UF support, as described by Maruf et al., [24, 25], were used. Permeation tests were performed in a crossflow filtration cell, allowing for better estimation of the boundary layer compared to the stirred cell used previously [24]. That is, the angle-of-attack between the bulk flow and the patterns were controlled in these experiments and the nominal mass transfer correlation for this cell had been elucidated in prior studies [27, 28]. The glycerol/ $\mathrm{NaCl} /$ water fractionation properties were evaluated using the solution-diffusion model-to assess whether patterning changes separation factors relative to the unpatterned counterparts, possibly due to a higher interfacial mass transfer coefficient than estimated from the classic Sherwood $(S h)$ correlation used-and the fouling effects were evaluated based on permeance decline, permeance recovery, and post-mortem adsorption characterization. In addition, we assess the variability in transport metrics within a single batch, and between 
batches, of these laboratory-scale membranes. At the outset, though, we also discuss how membrane compaction occurs over extended periods, and needs to be quantitatively assessed in order to draw more rational conclusions from subsequent fouling measurements.

\section{Materials and methods}

\subsection{Membrane fabrication}

Herein, "flat-TFC" refers to our TFC membranes fabricated on a flat, unaltered UF substrate, and, similarly, "NIL-TFC" refers to the composite membranes fabricated on nanoimprinted UF substrates. The substrate is a commercial polyethersulfone (PES) UF membrane (PW, GE Water and Infrastructure) with a nominal $30 \mathrm{~kg} / \mathrm{mol}$ molecular mass cutoff. The nanoimprinting process used for the UF membrane in this study was previously described in detail [18]. Briefly, the NIL process was carried out in an Eitrie 3 (Obducat, Inc.) nanoimprinter using a silicon mold containing parallel line-and-space gratings (a periodicity of $575 \mathrm{~nm}$, a line width of $210 \mathrm{~nm}$ and a groove depth of $180 \mathrm{~nm}$ ). The Si mold surface was treated with a Nanostrip ${ }^{\circledR}$ solution prior to the imprinting to clean off any debris from prior usage. The NIL process was carried out at $120^{\circ} \mathrm{C}$ and $4 \mathrm{MPa}$ for $180 \mathrm{~s}$, and the mold was separated from the membrane samples at $40^{\circ} \mathrm{C}$. The imprinted UF membranes were then cleaned and stored in de-ionized (DI) water in the dark prior to the interfacial polymerization (IP) step.

The first step of the IP process was to tape either the flat or imprinted UF substrate to a glass plate, with the skin layer facing out. Next, the substrate was immersed in an aqueous amine solution. This solution was prepared by adding $2 \mathrm{~g}$ of triethylamine (TEA, $99.5 \%$, Sigma Aldrich) and $4 \mathrm{~g}$ of (+)10-camphor sulfonic acid (CSA, $99.0 \%$, Sigma Aldrich) to $80 \mathrm{~mL}$ DI water with vigorous stirring. After the TEA-CSA mixture was completely dissolved, DI water was added to reach a total volume of $100 \mathrm{~mL}$. Next, $2 \mathrm{~g}$ of 1,3-phenylenediamine (MPD, Sigma Aldrich) was added to the TEA-CSA solution. The entire UF membrane was then immersed in the aqueous MPD-TEA-CSA solution for $8 \mathrm{~s}$, and the excess solution on the membrane surface was removed with an air blower. Subsequently, the amine-soaked UF membrane was immersed in a hexane solution (Fisher Scientific) containing $0.1 \%(\mathrm{w} / \mathrm{v})$ trimesoyl chloride (TMC, $99 \%$, Sigma-Aldrich) for $8 \mathrm{~s}$. The membrane was then removed from the hexane solution, cured at $70^{\circ} \mathrm{C}$ for $10 \mathrm{~min}$, and rinsed thoroughly with DI water. Finally, the as-prepared flat-TFC and NIL-TFC membranes were stored in DI water at $5{ }^{\circ} \mathrm{C}$ in the dark for 1-3 weeks. 


\subsection{Membrane characterization}

The surface topographies of the membranes, before and after fabrication processes, were examined with a field-emission scanning electron microscope (FESEM, Zeiss, Supra 60) and an atomic force microscope (AFM, Dimension 3100 AFM, Bruker). Membrane samples were dried in a vacuum oven and coated with a $\sim 4 \mathrm{~nm}$ gold layer prior to FESEM measurements. All AFM measurements were performed in "tapping" mode under ambient conditions using silicon cantilever probe tips (Veeco, RTESP) with spring constants ranging between 20 and $80 \mathrm{~N} / \mathrm{m}$ (nominal manufacturer specifications).

\subsection{Membrane filtration}

Each set of membranes was tested in triplicate. Experiments were conducted using the same 3cell membrane apparatus, experimental methods, and analytical protocols described previously [28]. Briefly, three membranes, each with a surface area of $9.6 \mathrm{~cm}^{2}$, were placed in this membrane module. A pump (Hydra-Cell, D/G-03 Series) was used to pressurize the solution from a single, $4 \mathrm{~L}$ feed, which was split into three streams upstream of the module, such that the three replicates were obtained simultaneously from the same feed solution in parallel. The imprinted membranes were installed such that the pattern grooves were perpendicular to the crossflow. Membranes were conditioned with DI water at $25^{\circ} \mathrm{C}$ at a transmembrane pressure of either 12 or 24 bar. Crossflow was provided at a rate of $0.26 \mathrm{~m} / \mathrm{s}\left(\operatorname{Re} \sim 10^{3}\right)$. Permeance was measured using a balance and timer, and is reported herein as the permeate flux per unit transmembrane pressure $\left(\mathrm{L} / \mathrm{m}^{2} / \mathrm{h} / \mathrm{MPa}\right.$, or $\left.\mathrm{LMH} / \mathrm{MPa}\right)$. Membrane conditioning was continued for up to 13 days until the pure water permeance (PWP) decreased less than 3\% over the previous $24 \mathrm{~h}$ period. Next, the feed was replaced with a solution of $0.14 \mathrm{M} \mathrm{NaCl}(\sim 8.2 \mathrm{~g} / \mathrm{L})$ and $0.014 \mathrm{M}$ glycerol $(1.3 \mathrm{~g} / \mathrm{L})$ in water, and the pressure was again increased to its initial value. The solution permeance was measured for $5 \mathrm{~h}$ to ensure its stability, and then samples were taken from the feed and permeate for later analysis. Next, a model protein foulant, BSA, was added to the feed at a concentration of $0.1 \mathrm{~g} / \mathrm{L}$, and the solution permeance was periodically measured. Samples were again taken from the feed and permeate after $2 \mathrm{~h}$. The permeance decline during BSA filtration was measured for $2 \mathrm{~h}$ for the membranes tested at $12 \mathrm{bar}$, and for $26 \mathrm{~h}$ for the 


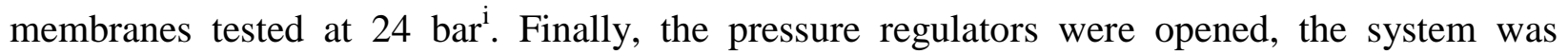
flushed three times with $4 \mathrm{~L}$ DI water (12 L total) at the same crossflow velocity as used in the filtration studies, the pressure regulators were reset to the initial pressure, and then the final pure water permeance was measured. The permeance recovery percentage is based on the PWP measured within $1 \mathrm{~h}$ of beginning the "final PWP" step. A schematic of the overall experimental filtration steps/protocol is provided in the Supplementary Information (SI).

Samples were later analyzed for their glycerol and $\mathrm{NaCl}$ concentrations using high-pressure liquid chromatography (HPLC) with a refractive index detector (Agilent 1100 Series). The 50- $\mu \mathrm{L}$ samples were injected into a hydrogen column (Phenomenex Rezex RHA), which was maintained at $60^{\circ} \mathrm{C}$. The mobile phase was degassed DI water with a flow rate of $0.6 \mathrm{~mL} / \mathrm{min}$. Concentrations were calculated using calibration curves, ensuring that measurements were taken within the linear response range between concentration and refractive index.

Transport was modeled using the solution-diffusion model [29]. Corrections for the concentration polarization boundary layer were made using a Sherwood correlation for laminar flow in a horizontal slit [30], consistent with the geometry of our membrane module [28]. More details are in the SI. The primary transport metric calculated herein is the separation factor, $\alpha_{i / j}$, and is calculated as the ratio between solution-diffusion permeance coefficients, $P$, for penetrants $i$ and $j$, i.e., $\alpha_{i / j}=P_{i} / P_{j}$. The penetrants include water $(w), \mathrm{NaCl}$ (electrolyte, $e$ ), and the glycerol (aka reduced carbon, $r$ ). If $\alpha_{i / j}<1$, then penetrant $i$ is less permeable than penetrant $j$, given the same activity driving force; if $\alpha_{i / j}>1$, then the converse is true. The filtration protocol is summarized in Table 1.

\footnotetext{
${ }^{\mathrm{i}}$ The low-pressure experiments were run for a shorter duration because minimal flux decline was observed over the time scale of the experiment. The back-pressure regulators were then modified to accommodate the higher pressures needed to reach a nominal $\mathrm{j} / \mathrm{k}>1$ for increased BSA deposition. More significant flux decline was observed at the higher pressure. Due to the extensive time required to manually prepare these membranes, the low-pressure experiments were not initially reproduced for the longer duration with the flat-TFC membranes.
} 
Table 1: Summary of the filtration protocol that was performed for three NIL-TFC and three flatTFC membranes at 12 and 24 bar.

\begin{tabular}{|l|l|}
\hline \multicolumn{1}{|c|}{ Feed composition } & \multicolumn{1}{c|}{ Transport metrics } \\
\hline DI water & $\begin{array}{l}\text { Initial PWP until compaction criterion met } \\
(<3 \% \text { change per day) }\end{array}$ \\
\hline $0.14 \mathrm{M} \mathrm{NaCl}, 0.014 \mathrm{M}$ glycerol, water & $\begin{array}{l}\text { Solution-diffusion permeance coefficients }\left(P_{i}\right), \\
\text { overall solution permeance }(\mathrm{LMH} / \mathrm{MPa})\end{array}$ \\
\hline $\begin{array}{l}1 \mathrm{~g} / \mathrm{L} \mathrm{BSA}, 0.14 \mathrm{M} \mathrm{NaCl}, 0.014 \mathrm{M} \text { glycerol, } \\
\text { water }\end{array}$ & $\begin{array}{l}\text { Solution-diffusion permeance coefficients }\left(P_{i}\right), \\
\text { overall solution permeance }(\mathrm{LMH} / \mathrm{MPa})\end{array}$ \\
\hline DI water & Final PWP \\
\hline
\end{tabular}

\subsection{Post-mortem adsorption and gravimetric measurements}

A post-mortem biochemical assay was used to characterize the protein associated with the membrane after permeation experiments [31]. After removing the membranes from the test cell, the membrane sample was sectioned into a $\sim 7 \mathrm{~cm}^{2}$ coupon. Next, the mass of each membrane coupon was measured using a high-resolution microbalance (Model ME235S, Sartorius). Watersoluble proteins were then eluted from the membrane coupons and their concentration measured using the following procedure: 1) the membrane coupon was aseptically placed in a $50 \mathrm{~mL}$ clean plastic test tube; 2) $15 \mathrm{~mL}$ of ultrapure sterile water was added, and the resulting solution was sonicated on ice for $1 \mathrm{~h}$; 3) the eluent was analyzed for protein content using a bicinchoninic acid kit and a BSA standard calibrator (Pierce); and 4) a spectrophotometer (model DR/2010, Hach) was used to measure the sample's absorbance at a wavelength of $562 \mathrm{~nm}$. The lower detection limit of this protein assay under the conditions used is $12 \mu \mathrm{g} / \mathrm{cm}^{2}$. The mass of the membrane coupon was again measured, and the percent increase in mass of the protein-fouled membranes compared to the sonicated membranes was calculated. Finally, the sonication protocol was repeated a second time to determine whether most of the protein on the membrane surface that could easily be removed via sonication was removed during the first iteration of the sonication protocol.

\section{Results and discussion}

\subsection{Membrane characterization}

AFM surface height profiles and FESEM images of the flat-TFC, NIL-UF, and NIL-TFC surfaces are shown in Figure 1. The flat-TFC membranes have random surface roughness with heights on the order of $10 \mathrm{~nm}$. The imprinted UF substrate has a regular, patterned surface with a 
$\sim 60 \mathrm{~nm}$ groove depth, while the groove depth of the polyamide thin-film, which was fabricated on top of the patterned UF substrate, has a lower, but still regular, groove depth of $\sim 30 \mathrm{~nm}$. Thus, the interfacial polymerization fills in some of the surface pattern of the UF membrane, that is, the interfacially polymerized layer is thicker within the grooves (crenels) than atop the lines (merlons) [25], but still results in a regularly patterned surface with larger protrusions as compared to the random roughness on the flat-TFC membrane.
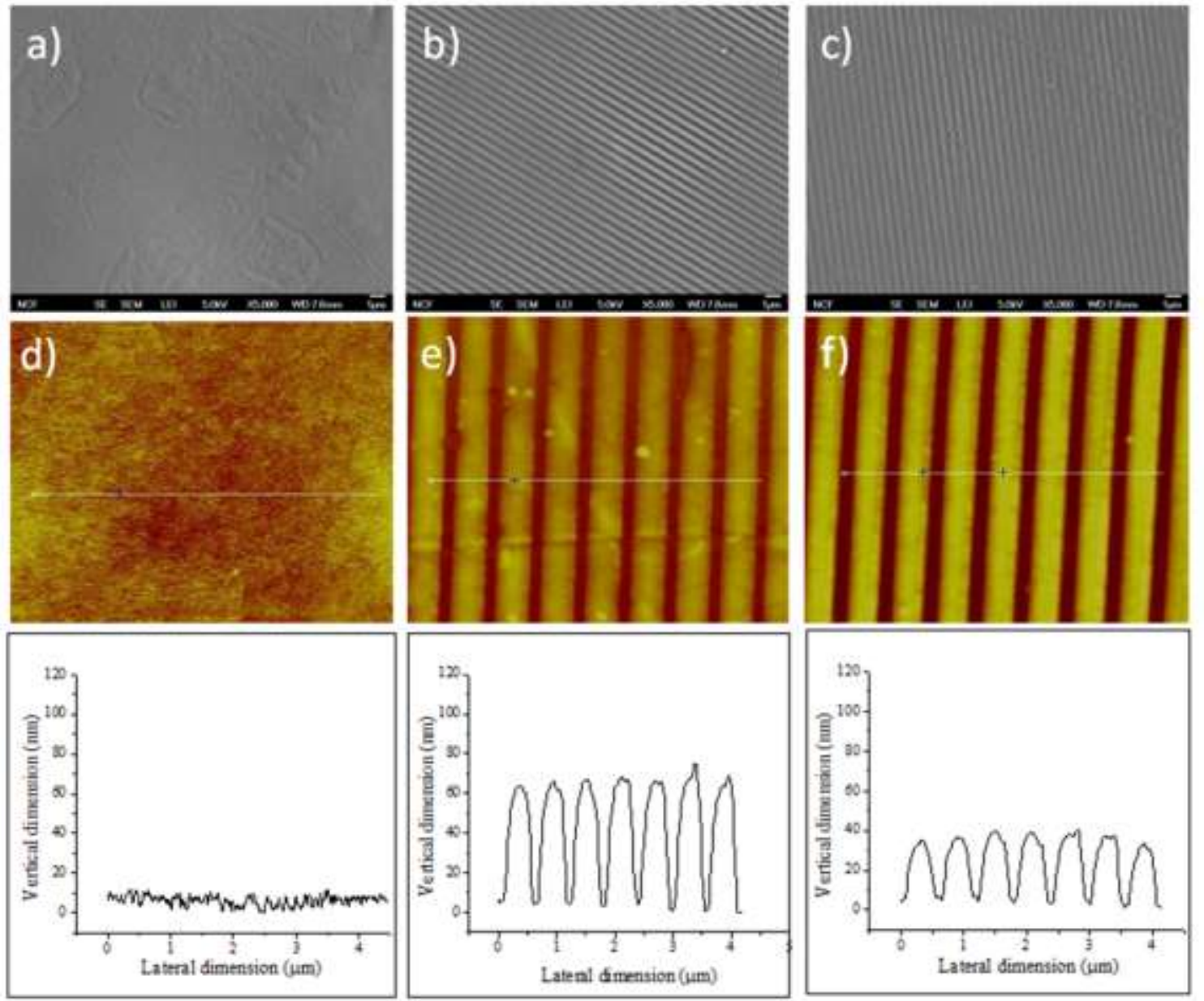

Figure 1: Morphological characterization of the flat-TFC, NIL-UF and NIL-TFC membranes. Representative top-surface SEM images of (a) flat-TFC membrane, (b) NIL-UF support membrane, (c) NIL-TFC membrane. Images (d), (e) and (f) are representative topographic AFM images of respectively flat-TFC, NIL-UF and NIL-TFC membranes obtained from AFM scans. The corresponding cross-sectional profiles are shown below the AFM images. 


\subsection{Membrane filtration experiments}

\subsubsection{Initial membrane conditioning}

Prior to filtering solutions, all membranes were first conditioned with DI water at a transmembrane pressure of either 12 bar or 24 bar. Figure 2 shows an example of the permeance measured for each of three NIL-TFC membrane replicates during the initial conditioning period at 24 bar. During this time, the pure water permeance (PWP) of all membranes decreases, likely due to substrate deformation (compaction). Note that the differences among the replicates are rather large. This finding is not surprising given the non-automated, manual techniques that were employed to fabricate the membranes. Despite their different initial permeances, each membrane displayed similar trends in permeance decline during compaction.

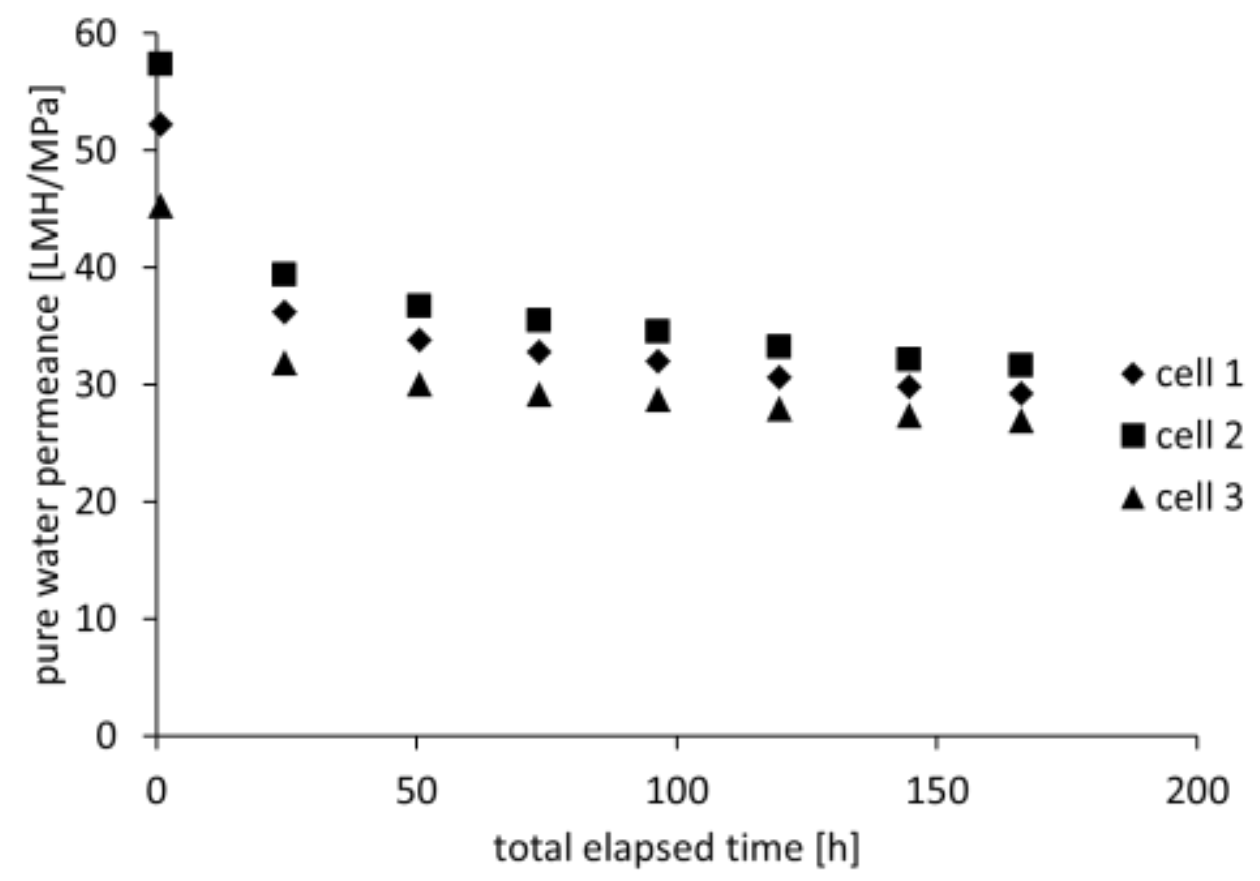

Figure 2: Permeance decline during initial conditioning of three NIL-TFC membrane replicates at 24 bar.

In order to present the data with minimal clutter, Figure 3 shows the mean values from the triplicate measurements, and $90 \%$ confidence intervals are included only for the final datum from each series. During this time, the PWP of all membranes decreases, likely due to compaction [32-38] (open diamond symbols in Figure 3). Although the NIL-TFC membrane permeance at 12 bar stabilizes after $\sim 3$ days, the permeance of the NIL-TFC at higher pressure 
and that of the flat-TFC at both pressures continues to decrease monotonically over longer times. This behavior of prolonged permeance decrease is also consistent with that of a commercial fully aromatic polyamide (FA-PA) membrane (ESPA1, Hydranautics, Figure 3c), and is mentioned in many studies of compaction, including [33, 36, 39] and references therein. Recently, Hussain and Al-Saleh [38] presented the idea that, since TFC membranes are composite structures, a VoigtWiechert mechanical model was more appropriate since it represents the structure as KelvinVoigt elements in series, each with its own relaxation time. Their analysis posits that flux decline occurs at a slower rate than the actual strain of the entire composite membrane because the flux decline in nominally localized within the thin, dense active layer, whose creep occurs over the longest time scale.

To accommodate the polymer deformation that occurs over long time scales, we set a criterion that there be less than 3\% decrease in PWP over the previous $24 \mathrm{~h}$ period for a membrane to be considered "stable." The NIL-TFC membrane likely stabilized more quickly than the flat-TFC membrane at 12 bar because the substrate has essentially been precompacted during the nanoimprinting process, which applies a pressure of $4.0 \mathrm{MPa}$ (40 bar) to the patterning mold/membrane at an elevated temperature $\left(120^{\circ} \mathrm{C}\right)$. The influence of precompaction was illustrated by Maruf et al. [25] when a flat-TFC membrane was created on a UF support that had been subjected to compression with a flat $\mathrm{Si}$ wafer under the same $\left(120^{\circ} \mathrm{C} / 4 \mathrm{MPa} / 180 \mathrm{~s}\right)$ conditions used for NIL. The permeance for the compressed support was $\sim 229 \mathrm{LMH} / \mathrm{MPa}$ and for the flat-TFC it was $\sim 55 \mathrm{LMH} / \mathrm{MPa}$, clearly showing that the TFC layer provides the majority of permeation resistance, as noted above. More significantly, there was essentially no difference in the short-time (2h) solution permeance listed in that work's Table 3 (or the flux decline in its Supplemental Information) between that flat-TFC and its counterpart NIL-TFC membrane, unlike the differences illustrated herein Figure $3 \mathrm{~d}$ and $3 \mathrm{e}$.

In general, increased PWP conditioning time and pressure appears to reduce the differences among replicates (for example, compare the confidence intervals in Figure $3 \mathrm{a}$ vs $3 \mathrm{~d}$, and Figure $3 \mathrm{~b}$ vs $3 \mathrm{e}$ ). This finding can be rationalized by reduced variability in the substrate structure as the largest macropores have deformed and the substrate becomes more compact.

Two cases of flat-TFC membranes are presented in Figure 3d. One case is wherein the membranes are the re-used ones from the results in Figure 3a. Since these flat-TFC membranes 
were initially tested at the lower pressure, and since the membrane permeance did not change significantly during the low-pressure experiment, the membranes were used again for the highpressure experiment. Subsequent conditioning at the higher pressure further reduced the PWP of these flat-TFC membranes, indicating that both the substrate and thin film layer continues to deform when it is subjected to a greater mechanical force. Note, that the permeance of the reused, flat-TFC membranes at 24 bar increased $2-5 \%$ at $\sim 115 \mathrm{~h}$. This increase corresponded to a power outage that turned off the pump, and hence reduced the pressure, and shows that the substrate deformation that occurs during membrane conditioning is at least partially recoverable. The second case presented in Figure $3 d$ is for a completely fresh set of flat-TFC membranes. These start out with a higher PWP and decline in the initial conditioning with a similar relaxation time-scale, but exhibit a higher PWP, than the re-used samples. This variation in final PWP is likely due to the longer overall conditioning time (and irreversible creep experienced by the composite structure) inherent from continuously using a membrane. In short, these results confirm that the reported PWP for a polymer membrane is highly sensitive to its history and that it is important to get to a quantifiable level of PWP change before commencing fouling assessment. 

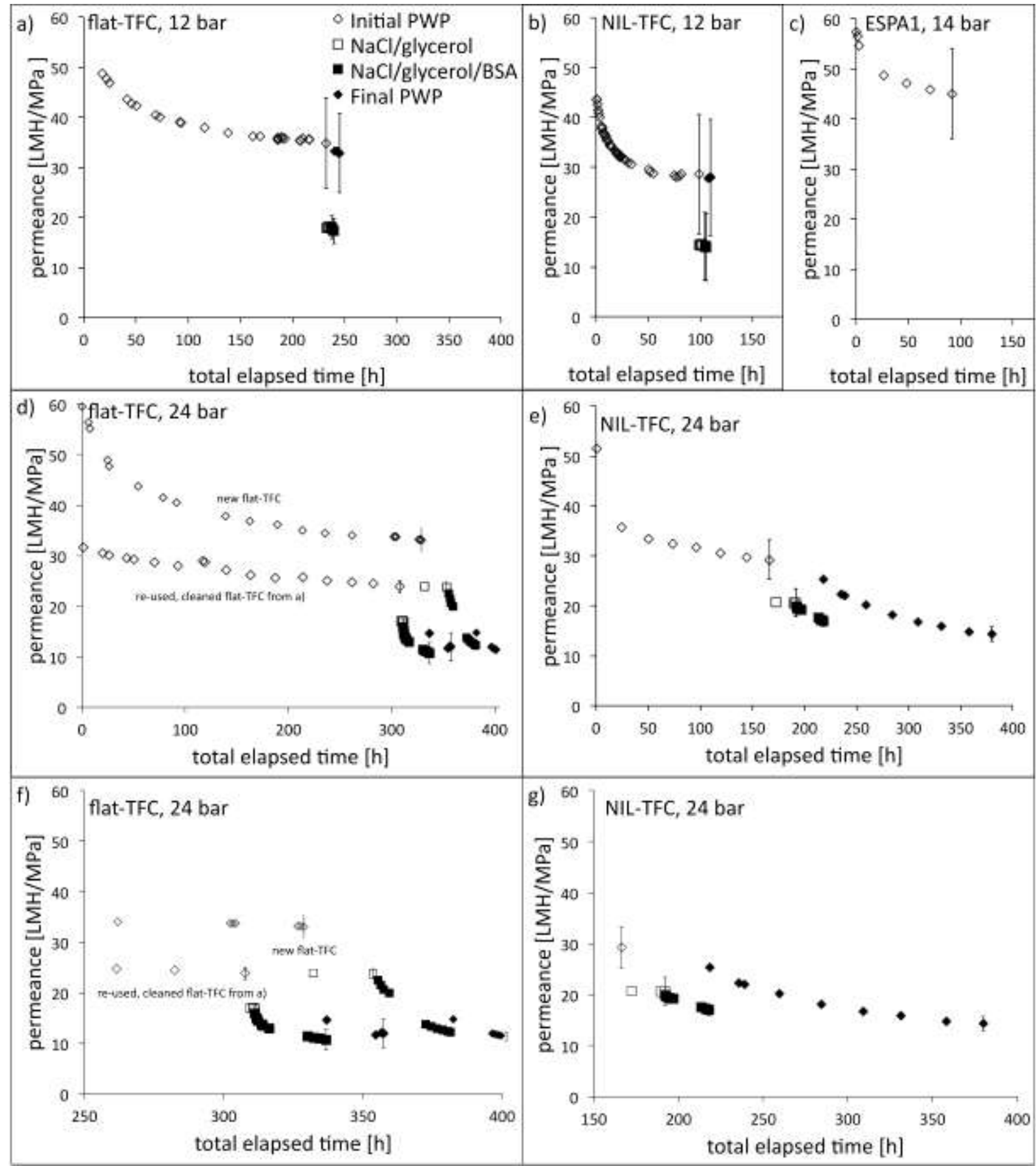

Figure 3: Pressure-normalized volumetric flux (permeance) during flat-TFC and NIL-TFC experiments at 12 bar (a and b) and 24 bar (d and e). Initial pure water permeance of ESPA1 (a commercial membrane) is also included (in c) to compare compaction behavior. Confidence bars are $90 \%$ confidence intervals for three membrane replicates. We only included confidence intervals for the last datum in each series to reduce clutter. In (d) we present two cases, one in which the flatTFC from (a) was reused, and one in which a completely new flat-TFC was used, similar to the cases of the NIL-TFC in (b) and (e). Note, for the new flat-TFC data in (d) the $90 \%$ confidence intervals for all but the inital PWP were contained within the plotting symbols' widths. Panels (f) and (g) focus in on the time scale of the permeance decline region for the data in (d) and (e), respectively. 
3.2.2. Fractionation properties during filtration of aqueous $\mathrm{NaCl} /$ glycerol, with and without BSA

After the PWP stabilized, the membranes' fractionation properties were measured for solutions containing water, $\mathrm{NaCl}$, and glycerol, with and without $\mathrm{BSA}$. The $\mathrm{NaCl}$ and glycerol true rejections (i.e., using the estimated concentration in the liquid at the feed-membrane interface, rather than the bulk concentration) for each membrane/pressure combination are given in Table 2. Due to the high glycerol and $\mathrm{NaCl}$ retention, we assumed that BSA was mostly retained ${ }^{\mathrm{ii}}$. A one-way analysis of variance (ANOVA) reveals that none of the species' permeance coefficients varies significantly ( $p=0.34,0.16$, and 0.52 for glycerol, $\mathrm{NaCl}$, and water, respectively $)^{\mathrm{iii}}$ for solutions with BSA versus those without it. Thus, we conclude that only minimal further mixture non-idealities were introduced by the addition of the BSA.

Table 2: $\mathrm{NaCl}$ and glycerol rejections for flat-TFC and NIL-TFC membranes, with and without BSA, at 12 bar and 24 bar. The \pm values are $90 \%$ confidence intervals for three membrane replicates.

\begin{tabular}{|c|c|c|c|c|c|}
\hline \multirow{2}{*}{} & \multicolumn{2}{c|}{$R_{\text {NaCl }}[\%]$} & \multicolumn{2}{c|}{$R_{\text {glycerol }}[\%]$} \\
\cline { 3 - 6 } & no BSA & + BSA & no BSA & + BSA \\
\hline \multirow{2}{*}{12 bar } & flat-TFC & $86 \pm 8$ & $87 \pm 7$ & $88 \pm 4$ & $89 \pm 3$ \\
\cline { 2 - 6 } & NIL-TFC & $90 \pm 4$ & $90 \pm 4$ & $92 \pm 5$ & $94 \pm 4$ \\
\hline \multirow{2}{*}{24 bar } & ${ }^{\dagger}$ flat-TFC & $95 \pm 2$ & $95 \pm 1$ & $95 \pm 1$ & $96 \pm 2$ \\
\cline { 2 - 6 } & NIL-TFC & $95 \pm 1$ & $96 \pm 1$ & $95 \pm 2$ & $96 \pm 2$ \\
\hline
\end{tabular}

'results are the average of both the re-used and new flat-TFC membranes' results

Figure 4 shows the separation factors (log scale) between water, glycerol, and $\mathrm{NaCl}$ for the NILTFC and flat-TFC membranes, together with other classes of polymer membranes that we characterized in a previous study [28]. Within the uncertainties of our measurements, imprinting the substrate does not substantially change the separation properties of the composite membrane. Thus, this factor should not influence the flux decline behavior. Also, we cannot observe any statistically significant differences in the separation factors between flat and NIL-TFC membranes. The variability within a batch of the flat-TFC and NIL-TFC membranes is similar to

\footnotetext{
${ }^{i i}$ BSA has a molecular mass of $66,463 \mathrm{~g} / \mathrm{mol}$, whereas that of glycerol is $92 \mathrm{~g} / \mathrm{mol}$. Thus, even if BSA had a higher solubility selectivity in the membrane than glycerol (unlikely), it would have a far lower mobility selectivity versus water, and therefore its rejection would be at least as high as that of glycerol.

iii A $p$-value of 0.34 indicates that there is only a $66 \%$ probability that the different mean values for a species' permeance with and without BSA in the mixture arise from different normal distributions. A maximum value of $p=$ 0.05 or 0.1 is usually used to test for statistical significance.
} 
that from a section of a roll of commercially available FA-PA membrane (ESPA 1). The flatTFC and NIL-TFC membranes fall within a similar but distinct region compared to commercial FA-PA membranes that we have also tested. Specifically, the water/glycerol separation factors are similar for both sets of membranes, but the membranes we fabricated are more permeable to $\mathrm{NaCl}$ vs glycerol, whereas the converse is true for the commercial membranes. Polymeric structural and chemical differences between our TFCs and the tested commercial membranes provide a likely rationale for this difference in $\alpha_{e / r}$, and further elucidation of the dense layer transport is beyond the scope of the current study. The variability between batches of the NILTFC membranes is on the order of the variability within any given batch of membranes.

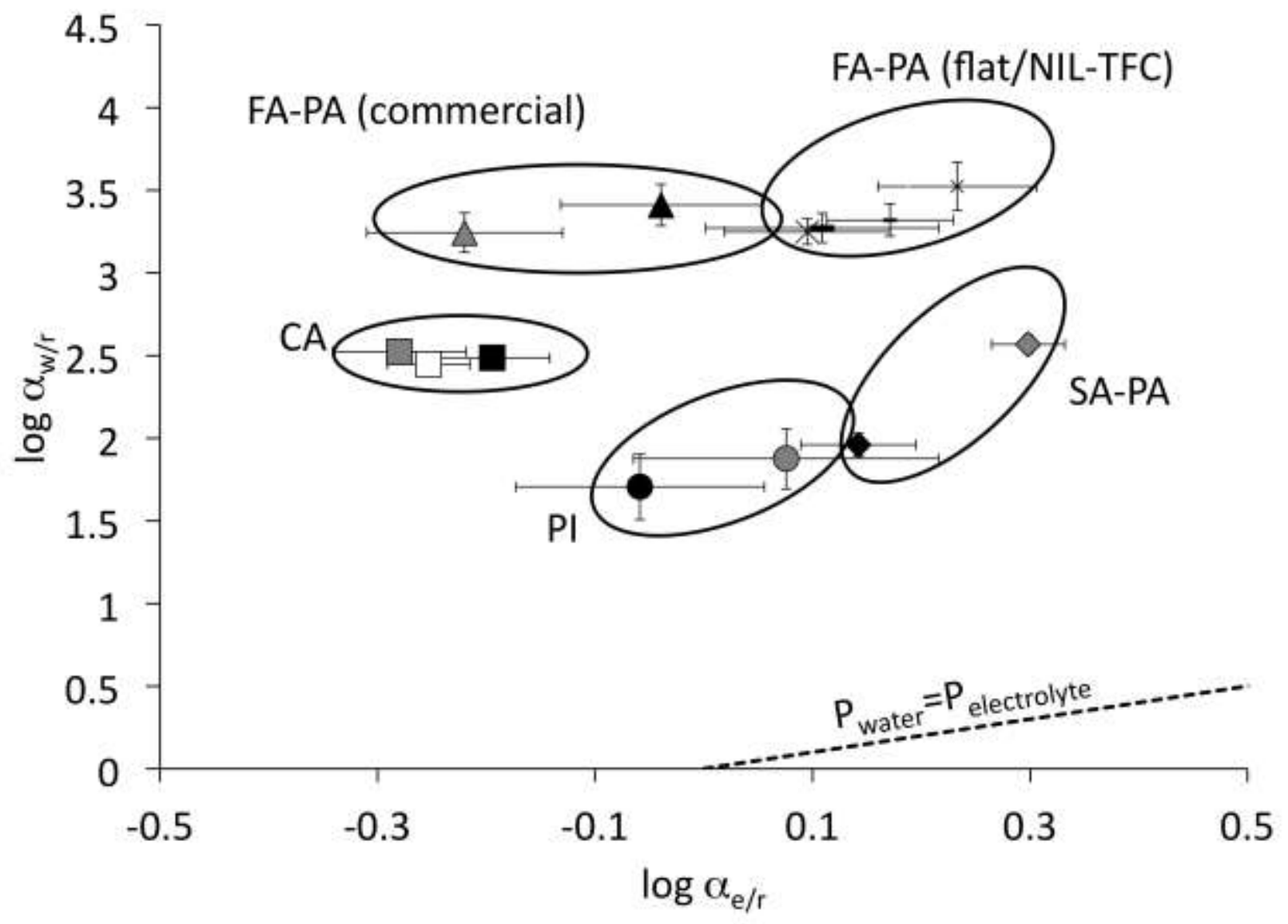

Figure 4: Separation factors between water (w), glycerol (r), and $\mathrm{NaCl}(\mathrm{e})$ for a variety of polymer material classes. Confidence bars are $\mathbf{9 0 \%}$ confidence intervals for three membrane replicates. Crosses are NIL-TFC; flat-bars are flat-TFC; and the size of the symbol correlates with lower (12 bar) or higher (24 bar) TMP used. Adapted from [28]: CA (cellulose acetate), PI (polyimide), SAPA (semi-aromatic polyamide), and FA-PA (fully-aromatic polyamide). 


\subsubsection{Permeance decline during BSA filtration}

After the permeance was measured for several hours to ensure its stability, a model protein foulant, BSA, was added to the feed with continued monitoring of the permeance for evidence of possible flux decline. Comparing the open versus filled squares for Figure 3a (flat-TFC) versus $3 \mathrm{~b}$ (NIL-TFC), we see hardly any difference when the flux is lower due to the $\Delta \mathrm{p}_{\mathrm{TM}}=12$ bar. Under these circumstances, there does not appear to be any fouling from addition of BSA. On the contrary, at the higher flux of $\Delta \mathrm{p}_{\mathrm{TM}}=24$ bar, we can compare the same open and filled squares in Figure $3 \mathrm{~d}$ and $\mathrm{f}$ (flat-TFC) and $3 \mathrm{e}$ and $\mathrm{g}$ (NIL-TFC) and notice that the rate of fouling is lower for the NIL-TFC, supporting the hypothesis of increased back mass transfer.

Due to the variability between the permeance of membranes in a single batch, the water permeance coefficient after BSA addition is normalized to the water permeance coefficient for the solution, prior to BSA addition and plotted in Figure 5. Note, that this is the water permeance, that is, the flux of water alone normalized by its activity gradient, which is different than the solution permeace (pressure gradient normalized total flux) plotted in Figure 3 (even though the same data is used in both figures). After $2 \mathrm{~h}$, the permeance of all membranes tested at the lower pressure reduces minimally, to $95-97 \%$ of its value before BSA addition for the flatTFC membranes, and $97-98 \%$ for the NIL-TFC membranes (Figure 5). This low permeance decline is likely due to operation with weak concentration polarization (CP) for BSA. A value of $J_{v} / k_{i}<1$, where $J_{v}$ is the volumetric flux and $k_{i}$ is the solute's mass transfer coefficient, indicates that there is weak CP, i.e., the rate of mass transfer of the solute back to the bulk is greater than (or equal to) its rate of convection toward the membrane, and deposition on the membrane is not expected [40]. For the experiments at 12 bar, operation is likely near the point for minimal/no BSA deposition, such that the slight decrease in water permeance is primarily from BSA adsorption. Note that we have already included osmotic composition effects in the activity coefficients used to calculate these permeances. The slightly greater permeance reduction for the flat-TFC membranes could be due to the higher initial permeance of that batch of membranes, leading to a higher value of $J_{v} / k_{i}$. In other words, at low TMP (flux) any possible effects from the membranes' different surface topologies are confounded with their different initial permeances. 


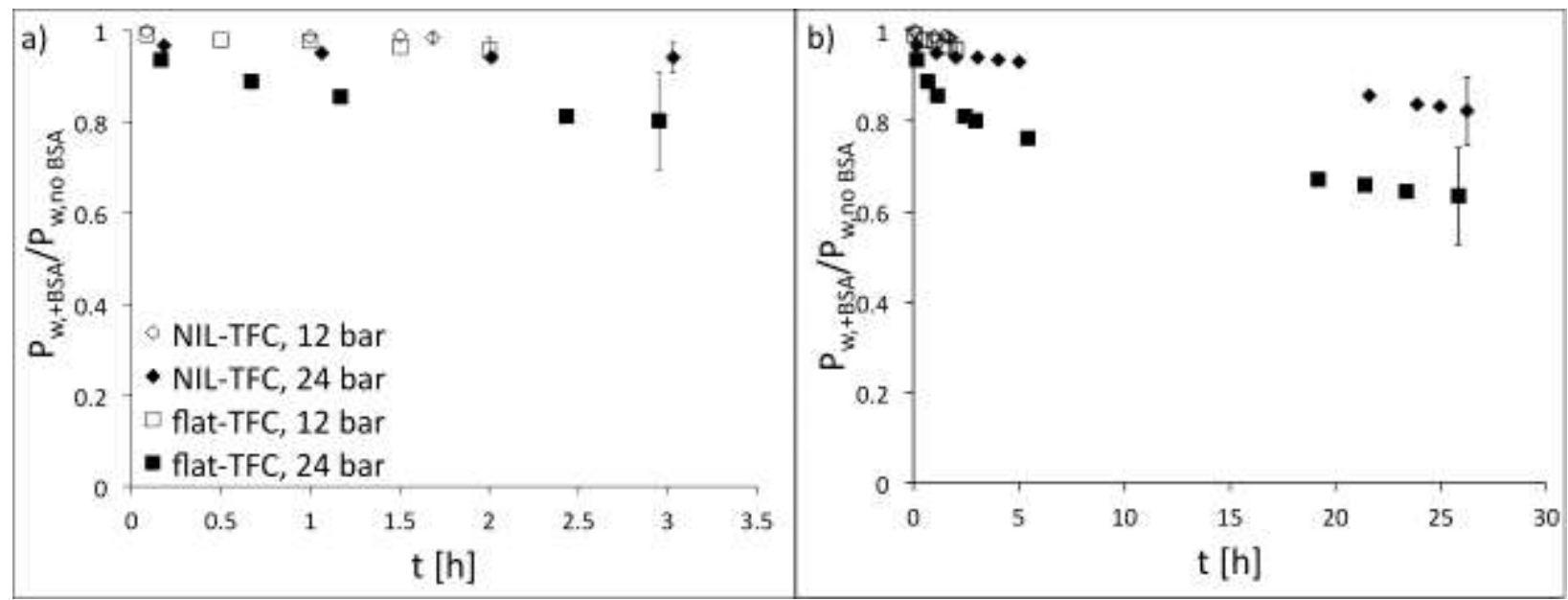

Figure 5: Water permeance coefficient during BSA fouling $\left(\mathbf{P}_{\mathrm{w},+\mathrm{BSA}}\right)$ normalized to water permeance coefficient prior to BSA addition ( $P_{w, n o}{ }_{B S A}$ ), as a function of time after BSA addition ( $\left.t\right)$, for NILTFC and flat-TFC membranes at 12 and 24 bar (open and filled symbols, respectively). Confidence bars are $90 \%$ confidence intervals for three membrane replicates. The LHS plot (a) employs an expanded scale to highlight the short time results.

Table 3. Volumetric flux divided by the calculated mass-transfer coefficient $\left(J_{v} / k_{i}\right)$ for $\mathrm{NaCl}$, glycerol, and BSA in flat-TFC and NIL-TFC membranes at 12 bar and 24 bar. The \pm values are $90 \%$ confidence intervals for three membrane replicates.

\begin{tabular}{|c|c|c|c|c|}
\hline \multicolumn{2}{|c|}{$J_{\Downarrow} / k_{i}$} & $\mathrm{NaCl}$ & glycerol & BSA \\
\hline \multirow{2}{*}{12 bar } & flat-TFC & $0.2 \pm 0.0$ & $0.2 \pm 0.0$ & $1.3 \pm 0.2$ \\
\cline { 2 - 5 } & NIL-TFC & $0.1 \pm 0.1$ & $0.2 \pm 0.1$ & $1.1 \pm 0.5$ \\
\hline \multirow{2}{*}{24 bar } & flat-TFC & $0.3 \pm 0.0$ & $0.4 \pm 0.0$ & $2.4 \pm 0.1$ \\
\cline { 2 - 5 } & NIL-TFC & $0.3 \pm 0.0$ & $0.5 \pm 0.1$ & $2.9 \pm 0.4$ \\
\hline
\end{tabular}

At the higher pressure, a greater initial decline in the normalized water permeance is observed for both membranes (Figure 5). However, despite the higher initial permeance of the NIL-TFC membranes, and thus higher $J_{v} / k_{i}$, the decrease in permeance is significantly less for the NILTFC than for the flat-TFC membranes ( $p=0.018$ for a paired t-test at the final point). The flatTFC membranes experience a rapid drop in permeance over the first few hours of operation, which then stabilizes to a more modest rate of permeance decline, similar to that of the NIL-TFC membranes. This initial decrease in permeance is likely associated with BSA deposition. As BSA is deposited, the permeance decreases until the CP is again low enough for adsorption (and possibly compaction of the fouling layer) to dominate. The greater permeance decline for the flat-TFC membranes suggests that the NIL-TFC membranes have improved hydrodynamics at the membrane-liquid interface, providing better disruption of the boundary layer and allowing for more advection away from the membrane surface. Permeance decline is still extant for the 
NIL-TFC membranes under these conditions, but there is a significant delay in its onset, such that $P_{w+B S A} / P_{w, n o B S A}=0.82$ was reached in $\sim 2.5 \mathrm{~h}$ for the flat-TFC membranes versus $26 \mathrm{~h}$ for the NIL-TFC membranes, despite the higher initial solution permeance for the latter (Figure 3).

\subsubsection{Permeance recovery after filtration}

After each permeation experiment with BSA solutions, the system was flushed with DI water (in simple crossflow with no extra applied pressure), and then the permeances of pure water were again measured ("Final PWP" in Figure 3). Table 4 summarizes the percentage of the initial PWP that was recovered after filtration, relative to the fouled membrane, defined as

$$
\text { permeance recovery }=\frac{\left(\begin{array}{ll}
P W P_{\text {cleaned }} & P W P_{\text {fouled }}
\end{array}\right)}{\left(\begin{array}{ll}
P W P_{\text {initial }} & P W P_{\text {fouled }}
\end{array}\right)} 100 \%
$$

Note that the permeance recovery percentage is based on the PWP measured within $1 \mathrm{~h}$ of beginning the "final PWP." Not surprisingly, the flat-TFC and NIL-TFC membranes that were operated at the lower pressure experienced little permeance decline, and recovered much of their initial permeance. The permeance recovery was slightly higher for the NIL-TFC membrane, but these membranes were also slightly less permeable than the flat-TFC, and so they may have experienced less of the already low BSA deposition. The estimated $J_{v} / k_{B S A}$ of the flat-TFC is 1.3 versus 1.1 for the NIL-TFC at the 12 bar condition (Table 3 ).

Table 4: Summary of permeance recoveries after BSA filtration and post-mortem characterizations. The \pm values are $\mathbf{9 0 \%}$ confidence intervals for three membrane replicates. The mass change compares the mass of the fouled membrane to the mass of the same membrane after sonication, and the protein concentration is that measured in the sonication supernatant.

\begin{tabular}{|c|c|c|c|c|}
\hline \multicolumn{2}{|c|}{} & permeance recovery [\%] & mass change [\%] & $\begin{array}{c}\text { protein } \\
\text { concentration } \\
{\left[\mu \mathrm{g} / \mathrm{cm}^{2}\right]}\end{array}$ \\
\hline \multirow{2}{*}{12 bar } & flat-TFC & $89 \pm 2$ & n/a & n/a \\
\cline { 2 - 5 } & NIL-TFC & $96 \pm 1$ & $0.1 \pm 0.1$ & $98 \pm 2$ \\
\hline \multirow{2}{*}{24 bar } & flat-TFC & $30 \pm 8$ & $0.7 \pm 0.6$ & $111 \pm 1$ \\
\cline { 2 - 5 } & NIL-TFC & $69 \pm 9$ & $0.3 \pm 0.1$ & $104 \pm 10$ \\
\hline
\end{tabular}

The membranes operated at 24 bar recovered much less of their initial permeance. Nonetheless, the NIL-TFC membranes have $\sim 2 \mathrm{X}$ the permeance recovery compared to the flat-TFC membranes, consistent with their improved permeance during BSA filtration. After continuing the pure water permeation experiments for $17 \mathrm{~h}$, there are surprising $\sim 20 \%$ and $12 \%$ drops in 
permeance for the flat-TFC and NIL-TFC membranes, respectively. We continued to measure the PWP of the NIL-TFC membranes for 7 days. During this time, the permeance declined rapidly before reaching a more stable value of $4 \%$ decrease per day. The drop in permeance takes place at a much faster rate than what we would expect if it was simply caused by compaction of the membrane itself, for which we had already set the permeance decline criterion of $<3 \% /$ day before starting the mixture filtration protocol.

The long-term decline in the permeance of fouled NIL-TFC membranes at 24 bar (Figure 3e and g) appears to be caused by compaction of the deposited protein layer. (Though, we note the possible confounding influence that may be related to slow $\mathrm{NaCl}$ removal, and the electrostatic shielding it can provide against membrane creep, as discussed by Nilsson et al. [41].) The postmortem protein analysis (Table 4) confirms that BSA is present on the membranes after the final PWP. This protein was likely adsorbed to the membrane surface such that it was not washed away during the post-filtration flushing or PWP. Note, that the protein concentration represents only the protein that was removed by the sonication protocol. After the first sonication, this protocol was repeated, and the amount of protein that could be removed during the second sonication was $16 \mu \mathrm{g} / \mathrm{cm}^{2}$ for each set of membranes. For reference, this value is only slightly higher than the lower detection limit of the bioassay under the conditions used $\left(12 \mu \mathrm{g} / \mathrm{cm}^{2}\right)$. Thus, it appears that most of the protein that could be easily removed using sonication is removed in the first hour of sonication.

The post-mortem analysis in Table 4 shows that the membranes with reduced permeance recovery also had more material removed during sonication and a higher protein concentration in the sonication supernatant. This protein concentration only represents the adsorbed protein that could not be removed by the shear from crossflow in the membrane module, yet could be removed by the sonication protocol. Thus, this analysis provides support for the hypothesis that a compact protein layer is present on the surface of the membrane. This hypothesis is also consistent with recent work on BSA membrane fouling in which the authors reported the formation of a compressible protein layer [42]. Furthermore, our results suggest that the protein layer on the NIL-TFC membranes has a more open structure than that on the flat-TFC membrane, such that the former was more easily removed by simple crossflow while the latter requires more vigorous methods (i.e., sonication) for protein removal. 


\section{Concluding remarks}

With weak concentration polarization, the difference between flat-TFC and NIL-TFC membranes is not significant, at least in our relatively short-time flux-decline experiments. No differences in the glycerol/ $\mathrm{NaCl} /$ water fractionation properties of the flat-TFC and NIL-TFC membranes could be distinguished, and the variability between batches is on the order of that within a batch. At higher flux, and more $\mathrm{CP}$, the imprinted membranes have less permeance decline and greater permeance recovery with simple flushing compared to their flat counterparts. Notably, at the higher pressure and at the conditions investigated, the NIL-TFC membranes can operate for ten times longer than the flat-TFC membranes before their permeance declines to $82 \%$ of the initial value. This suggests that imprinted materials can be operated for longer duration between cleanings. After $26 \mathrm{~h}$ of BSA filtration, the NIL-TFC membranes recovered $25 \%$ more of their initial permeance compared to the flat-TFC membranes, after simple water flushing. Our results suggest that, although protein accumulates on both flat and imprinted NF membranes, its rate of deposition may be slowed on the NIL-TFC membrane due to improved local hydrodynamics caused by the regular surface patterning. These features and the local shear environment may also result in a less dense protein layer, which is easier to remove with the shear provided during crossflow filtration compared to the protein layer on the flat surface. In summary, the imprinted membranes offer a means to increase operating time between cleanings and possibly also increase membrane lifetime without compromising the transport properties of the material, when using thin-film polymerization techniques that are already broadly utilized.

\section{Acknowledgements}

The authors acknowledge the funding support from the National Science Foundation under Grant No. CBET-1031785. The authors gratefully acknowledge the National Science Foundation (NSF) Industry/University Cooperative Research Center for Membrane Science, Engineering and Technology (MAST) at the University of Colorado at Boulder (CU-B) for their support of this research via NSF Award IIP 1034720. We also thank the U.S. Department of Education for providing a Graduate Assistance in Areas of National Need (GAANN) fellowship to Melissa Rickman. 


\section{References}

[1] H.J. Huang, S. Ramaswamy, U.W. Tschierner, B.V. Ramarao, A review of separation technologies in current and future biorefineries, Separation and Purification Technology, 62 (2008) 1-21.

[2] A.K. Gautam, T.J. Menkhaus, Performance evaluation and fouling analysis for reverse osmosis and nanofiltration membranes during processing of lignocellulosic biomass hydrolysate, Journal of Membrane Science, 451 (2014) 252-265.

[3] K.R. Colyar, J. Pellegrino, K. Kadam, Fractionation of pre-hydrolysis products from lignocellulosic biomass by an ultrafiltration ceramic tubular membrane, Separation Science and Technology, 43 (2008) 447-476.

[4] H. Wang, H. Zhou, Understand the basics of membrane filtration, in: Chemical Engineering Progress, American Institute of Chemical Engineers, New York, 2013, pp. 33-40.

[5] S.G. Yiantsios, D. Sioutopoulos, A.J. Karabelas, Colloidal fouling of RO membranes: an overview of key issues and efforts to develop improved prediction techniques, Desalination, 183 (2005) 257-272.

[6] J. Cho, G. Amy, J. Pellegrino, Y. Yoon, Characterization of clean and natural organic matter (NOM) fouled NF and UF membranes, and foulants characterization, Desalination, 118 (1998) 101-108.

[7] H. Huiting, J.W.N.M. Kappelhof, T.G.J. Bosklopper, Operation of NF/RO plants: from reactive to proactive, Desalination, 139 (2001) 183-189.

[8] M.L. Carman, T.G. Estes, A.W. Feinberg, J.F. Schumacher, W. Wilkerson, L.H. Wilson, M.E. Callow, J.A. Callow, A.B. Brennan, Engineered antifouling microtopographies - correlating wettability with cell attachment, Biofouling: The Journal of Bioadhesion and Biofilm Research, 22 (2006) 11-21. [9] J.F. Schumacher, M.L. Carman, T.G. Estes, A.W. Feinberg, L.H. Wilson, M.E. Callow, J.A. Callow, J.A. Finlay, A.B. Brennan, Engineered antifouling microtopographies - effect of feature size, geometry, and roughness on settlement of zoospores of the green alga Ulva, Biofouling, 23 (2007) 55-62.

[10] C.J. Long, J.F. Schumacher, P.A.C. Robinson, J.A. Finlay, M.E. Callow, J.A. Callow, A.B. Brennan, A model that predicts the attachment behavior of Ulva linza zoospores on surface topography, Biofouling, 26 (2010) 411-419.

[11] S.T. Reddy, K.K. Chung, C.J. McDaniel, R.O. Darouiche, J. Landman, A.B. Brennan, Micropatterned surfaces for reducing the risk of catheter-associated urinary tract infection: an in-vitro study on the effect of Sharklet micropatterned surfaces to inhibit bacterial colonization and migration of uropathogenic Escherichia Coli., Journal of Endourology, 25 (2011) 1547-1552.

[12] J.T. Decker, C.M. Kirschner, C.J. Long, J.A. Finlay, M.E. Callow, J.A. Callow, A.B. Brennan, Engineered antifouling microtopographies: an energetic model that predicts cell attachment, Langmuir, 29 (2013) 13023-13030.

[13] R.M. May, C.M. Magin, E.E. Mann, M.C. Drinker, J.C. Fraser, C.A. Siedlecki, A.B. Brennan, S.T. Reddy, An engineered micropattern to reduce bacterial colonization, platelet adhesion and fibrin sheath formation for improved biocompatibility of central venous catheters, Clinical and translational medicine, 4 (2015) 9-9.

[14] Y.X. Lu, J.Q. Sun, J.C. Shen, Cell adhesion properties of patterned poly(acrylic acid)/poly(allylamine hydrochloride) multilayer films created by room-temperature imprinting technique, Langmuir, 24 (2008) 8050-8055.

[15] D. Perera-Costa, J.M. Bruque, M.L. Gonzalez-Martin, A.C. Gomez-Garcia, V. Vadillo-Rodriguez, Studying the influence of surface topography on bacterial adhesion using spatially organized microtopographic surface patterns, Langmuir, 30 (2014) 4633-4641.

[16] S.H. Maruf, M. Rickman, W. L., J. Mersch IV, A.R. Greenberg, J. Pellegrino, Y. Ding, Influence of sub-micron surface patterns on the deposition of model proteins during active filtration, Journal of Membrane Science, 444 (2013) 420-428.

[17] S.H. Maruf, A.R. Greenberg, J. Pellegrino, Y. Ding, Critical flux of surface-patterned ultrafiltration membranes during cross-flow filtration of colloidal particles, Journal of Membrane Science, 471 (2014) 65-71. 
[18] S.H. Maruf, L. Wang, A.R. Greenberg, J. Pellegrino, Y. Ding, Use of nanoimprinted surface patterns to mitigate colloidal deposition on ultrafiltration membranes, Journal of Membrane Science, 428 (2013) 598-607.

[19] C.Y.Y. Tang, Y.N. Kwon, J.O. Leckie, Effect of membrane chemistry and coating layer on physiochemical properties of thin film composite polyamide RO and NF membranes II. Membrane physiochemical properties and their dependence on polyamide and coating layers, Desalination, 242 (2009) 168-182.

[20] G.Y. Chai, W.B. Krantz, Formation and characterization of polyamide membranes via interfacial polymerization, Journal of Membrane Science, 93 (1994) 175-192.

[21] V. Freger, Nanoscale heterogeneity of polyamide membranes formed by interfacial polymerization, Langmuir, 19 (2003) 4791-4797.

[22] V. Freger, Kinetics of film formation by interfacial polycondensation, Langmuir, 21 (2005) 18841894.

[23] G.Z. Ramon, E.M.V. Hoek, Transport through composite membranes, part 2: Impacts of roughness on permeability and fouling, Journal of Membrane Science, 425-426 (2013) 141-148.

[24] S.H. Maruf, A.R. Greenberg, J. Pellegrino, Y. Ding, Fabrication and characterization of a surfacepatterned thin film composite membrane, Journal of Membrane Science, 452 (2014) 11-19.

[25] S.H. Maruf, A.R. Greenberg, Y. Ding, Influence of substrate processing and interfacial polymerization conditions on the surface topography and permselective properties of surface-patterned thin-film composite membranes, Journal of Membrane Science, 512 (2016) 50-60.

[26] S.T. Weinman, S.M. Husson, Influence of chemical coating combined with nanopatterning on alginate fouling during nanofiltration, Journal of Membrane Science, 513 (2016) 146-154.

[27] M. Rickman, R.H. Davis, J. Pellegrino, Temperature-variation study of neutral solute and electrolyte fractionation through cellulose acetate and polyamide membranes, Journal of Membrane Science, 461

(2014) 114-122.

[28] M. Rickman, R.H. Davis, J. Pellegrino, Fractionation of organic fuel precursors from electrolytes with membranes, Industrial \& Engineering Chemistry Research, 52 (2013) 10530-10539.

[29] J.G. Wijmans, The solution-diffusion model: a review, Journal of Membrane Science, 107 (1995) 121.

[30] E.L. Cussler, Diffusion: Mass Transfer in Fluid Systems, 2nd Ed., Cambridge University Press, New York, 1997.

[31] E. Kujundzic, A.R. Greenberg, R. Fong, B. Moore, D. Kujundzic, M. Hernandez, Biofouling potential of industrial fermentation broth components during microfiltration, Journal of Membrane Science, 349 (2010) 44-55.

[32] J.L. Bert, Membrane compaction - a theoretical and experimental explanation, Journal of Polymer Science Part B-Polymer Letters, 7 (1969) 685-\&.

[33] H. Ohya, Expression method of compaction effects on reverse-osmosis membranes at high-pressure operation, Desalination, 26 (1978) 163-174.

[34] Y. Kurokawa, M. Kurashige, N. Yui, A viscoelastic model for initial flux decline through reverseosmosis membrane, Desalination, 52 (1984) 9-14.

[35] K.W. Lawson, M.S. Hall, D.R. Lloyd, Compaction of microporous membranes used in membrane distillation .1. effect on gas-permeability, Journal of Membrane Science, 101 (1995) 99-108.

[36] M.T.M. Pendergast, J.M. Nygaard, A.K. Ghosh, E.M.V. Hoek, Using nanocomposite materials technology to understand and control reverse osmosis membrane compaction, Desalination, 261 (2010) 255-263.

[37] V.R. Tarnawski, P. Jelen, Estimation of compaction and fouling effects during membrane processing of cottage cheese whey, Journal of Food Engineering, 5 (1986) 75-90.

[38] Y.A. Hussain, M.H. Al-Saleh, A viscoelastic-based model for TFC membranes flux reduction during compaction, Desalination, 344 (2014) 362-370. 
[39] R.A. Peterson, A.R. Greenberg, L.J. Bond, W.B. Krantz, Use of ultrasonic TDR for real-time noninvasive measurement of compressive strain during membrane compaction, Desalination, 116 (1998) 115-122.

[40] P. Bacchin, P. Aimar, R.W. Field, Critical and sustainable fluxes: Theory, experiments and applications, Journal of Membrane Science, 281 (2006) 42-69.

[41] M. Nilsson, G. Tragardh, K. Ostergren, The influence of sodium chloride on mass transfer in a polyamide nanofiltration membrane at elevated temperatures, Journal of Membrane Science, 280 (2006) 928-936.

[42] Y.N. Wang, C.T. Tang, Protein fouling of nanofiltration, reverse osmosis, and ultrafiltration membranes-The role of hydrodynamic conditions, solution chemistry, and membrane properties, Journal of Membrane Science, 376 (2011) 275-282. 